\title{
Evaluation of Retinopathy of Prematurity in Preterm Infants
}

\author{
Research Article
}

\section{Seyed Ahmad Rasoulinejad ${ }^{\text {* }}$}

1. Department of Ophthalmology, Associate Professor of Retina \& Vitreous, School of Medicine Social Determinants of Health Research Center, Ayatollah Rouhani Hospital, Babol University of Medical Sciences, Babol, Iran.

\begin{abstract}
Background: Retinopathy of Prematurity is aberrant developmental retinal Angiogenesis in preterm infants. For ophthalmologist diagnosis and management of this progressive disease remains a challenge. Objective: To investigate the retinopathy of prematurity in premature infants referred to the Eye Clinic of Ayatollah Rouhani Hospital in Babol in 2016. Methods: In this cross-sectional study, data on premature infants including risk factors for retinopathy and eye examinations were recorded and data were analyzed using Chi-square, single, and multiple logistic regression. Results: Among 100 premature infants with mean gestational age, 31.83 (GA) weeks (2. 06SD) and birth weight $1686.9(\mathrm{~g})(416.4 \mathrm{SD})$, retinopathy was $39 \%$ and severe in $14 \%$ of all specimens. In singlevariable analysis, there was a significant relationship between Retinopathy of Prematurity and birth weight, gestational age, duration of oxygen therapy, duration of hospitalization and sepsis, while in multivariate logistic regression, birth weight and respiratory distress syndrome were independent predictors of retinopathy. 25 cases (64. 1\%) had spontaneous regression and in 14 cases $(35.8 \%)$ treatment was required. The prognosis of treatment was desirable in all 14 cases. Conclusion: Timely screening and treatment in infants with severe retinopathy is very important in preventing progression of the disease to higher stages and poor vision prognosis.
\end{abstract}

\section{Keywords: Retinopathy of Prematurity, Premature Infant, Vision Prognosis, Risk Factors.}

\section{Introduction}

Retinopathy of Prematurity (ROP) is a vascular disorder that occurs in premature infant whose retinas is not yet fully vascularized. This disease occurs due to the occurrence of a damaging agent in the course of completing retinal vascularization, which can lead to the appearance of fibro-vascular tissue and its penetration into the vitreous and eventually blindness of the baby (1). Prevention of blindness in children under the age of one is the World Health Organization's plans by 2020 (2). Retinopathy is one of the potentially preventable causes of blindness in children and is responsible for 50,000childhood blindness each year $(3,4)$. Retinopathy of Prematurity is one of the most important causes of blindness in countries where the mortality rate for children under the age of one is 10-60 per 1000 live births (5). The disease, if promptly diagnosed, provides a good response to treatment, but delay in diagnosis and treatment shortly leads to blindness, various methods for the classification, diagnosis and treatment of ROP are provided (6). In recent years, with the increase in the survival of very low birth weight infants (VLBW) due to the

*Corresponding Author:

Seyed Ahmad Rasoulinejad,

Department of Ophthalmology,

Associate Professor of Retina \& Vitreous,

School of Medicine Social Determinants of Health

Research Center,

Ayatollah Rouhani Hospital,

Babol University of Medical Sciences, Babol, IRAN. progression of the NICU, the proportion of high-risk infants has increased for the development of ROP (7). The most important risk factors associated with ROP are low gestational age (GA) and low birth weight (BW) (8), so that the ROP is inversely proportional to gestational age birth weight (9). Other factors include sepsis, oxygen uptake, NICU hospitalization time, exposure to steroids, and mechanical ventilation for more than 7 days, exchange of blood and receiving surfactant $(10,11$, and 12).

In a recent study, in $70 \%$ infants with a birth weight of less than $1250 \mathrm{~g}$ and advanced ROP, treatment for severe type of disease was needed in only $6 \%$ of cases (13). In premature infants, the growth and development of retinal blood vessels is not yet completely understood. Perhaps the pathogenesis of ROP is that prematurity interferes with the process of natural retinal vascularization. Retinal growth in postnatal period occurs in an unstable and high-oxygen environment, and the effect of hypoxia-induced stimulation on the development of retinal vascular system decreases. The result is delayed vascularization of the retina, which ultimately leads to abnormal vessels and neovascularization. The problem with abnormal blood vessels is that they do not give enough oxygen to the retina. Retinopathy is divided into 5 stages and 3 zones. The progression of the disease to the last degree can cause retinal complications, such as retinal detachment, vitreous hemorrhage, and blindness.

The likelihood of survival in premature infants has increased in recent years in countries such as Iran, with an increase in the general level of health and treatment and a reduction in the mortality rate of infants, and it is expected that Iran is one of the countries that is 
at high risk of severe visual impairment caused by ROP (14). Although a large group of infants with ROP undergo spontaneous regression without any complications, approximately $6 \%$ of low-birth weight infants (less than $1251 \mathrm{~g}$ ) require advanced treatment for severe ROP. Despite the advances in prevention, detection and treatment of ROP in the world, complications such as cataracts, glaucoma and amblyopia have increased the complexity of the problem. Considering the importance of early diagnosis and treatment of ROP optimal vision prognosis in infants develops, in this study, we examined the ROP course and the prognosis of treatment.

\section{Preventive retinopathy screening}

Evaluation of the screening program involves performing a complete eye examination by a qualified ophthalmologist in neonatal ophthalmology. The pupils should be dilated to see the vitreous and retina. It is recommended that droplets containing low phenylephrine and Cyclopentolate concentrations be used 30 minutes before the eye examination with binocular indirect ophthalmoscope using 28D lens by expertise ophthalmologist (15). Using eye drops for pupillary dilation and eye manipulation can cause systemic complications for the baby, most of all are bradycardia and cardiac arrhythmia. Therefore, accurate monitoring of the baby is essential during the examination. An examiner can also use topical anesthetics (16). Initial screening tests are performed 4 to 6 weeks after birth. Compared to GA, for infants born at 22-26 weeks of gestation, initial examinations are performed at week 30 and for babies born at weeks 27 or over at 4 weeks postnatal. This program meets the criteria for screening in developed countries for infants weighing less than or equal to $1250 \mathrm{~g}$ or a gestational age of less than 31 weeks, obtained using 2 randomized trials $(17,18)$.

\section{Research Questions} ROP?

What are the factors influencing the incidence of

How many of the studied samples progress to the higher stages of the disease?

\section{Methodology}

The present study was conducted to investigate the retinopathy of prematurity in premature infants referred to the Eye Clinic of Ayatollah Rouhani Hospital in Babol in 2016. The study was a cross-sectional study. The target population included all premature infants referred to the NICU department (north of the Iran). The sample population was selected from the target community with the requirement to enter the study. Thus, by examining the case records of the infants, the following two groups were studied as target society:

1- Preterm infants with gestational age less than 32 weeks or birth weight less than 1500 grams discharged from the NICU even though they have not received treatment with oxygen.

2- Premature infants between 1500-2500 grams or more than 32 weeks have an unstable clinical course and are admitted to the NICU.
After considering the conditions of entry and exit, 106 newborns were enrolled in the study, of which were excluded five newborns were excluded due to lack of referral after the initial examination and one newborn due to lack of referral for treatment follow-up treatment was with $0 / 675 \mathrm{mg}$ Avastin injected intravitreally. Finally 100 newborns were examined and followed up. Sampling method was simple non-random. The patient information summary is extracted and entered in the questionnaire that is included. This information includes gestational age, birth weight, gender, oxygen therapy, type of oxygen therapy, hospitalization period, oxygen therapy period, and underlying disease. Four quadrants of each of the two eyes were subjected to precise examination, and then the stage and zone were recorded according to the international classification of Retinopathy of Prematurity (ICROP) in the questionnaire form. Follow-up examinations were performed every 2-3 weeks, once a week or twice weekly, in infants weighing less than 1000 grams or premature infants with active obesity retinopathy. In the case of retinopathy, THRESHOLD ROP(T-ROP) was immediately treated in less than 72 hours and follow-up examinations were carried out once a week in the first month, then every month (depending on the severity of the disease) to determine the prognosis of the treatment.

All information was entered into SPSS software version 15 and then analyzed. Descriptive statistical methods such as Frequency and Descriptive for examining the percentage of frequency of clinical and preclinical manifestations, chi-square test was used for analyzing qualitative variables and t-test was used for analyzing the quantitative variables and the logistic regression test was used to determine the relationship between variables with pre-term retinopathy.

\section{Findings}

In the study of premature infants referred to the NICU section of different treatment centers (north of Iran) in the Eye Clinic of Ayatollah Rouhani Hospital in Babol in 2016, the findings and the following results were obtained:

Of the 100 infants, 58 infants (58\%) were male and 42 infants $(42 \%)$ were female. The mean gestational age of the subjects (GA) was $31.83 \pm 2$. 06 weeks, with a minimum of 26 and a maximum of 36 weeks. The mean birth weight (BW) of the study group was $1686.9 \pm 416$. 47 grams, which weighed at least 630grams and a maximum of 2700 grams. 79 infants were treated with supplemental oxygen; among which oxy hood (53\%) was the most commonly used method for supplemental oxygen therapy. The mean duration of oxygen therapy was $4.5 \pm 4.6$ days. The mean hospitalization time was $18.75 \pm 13.76$ with a minimum of 2 and a maximum of 90 days.

ROP was confirmed in $39 \%$ of the samples, of which 14 (35. 8\%) had therapy after the first examination (T-ROP). Of the cases with retinopathy, 8 cases (20. 5\%) had Stage 1 disease, 17 cases (43. 5\%) had Stage 2 disease, and 14 cases (36\%) had Stage 3 disease. In the zone involved, 25 cases $(25 \%)$ of the zone 1 and 14 (14\%) were affected by zone 2 disease, and in no case retinopathy occurred in zone 3 . The 
highest prevalence of ROP was in the range of 29-32 weeks of gestational age (GA). ROP was seen in 29 cases of less than or equal to 32 weeks, and in 10 cases more than 32 weeks old. The relationship between ROP and gestational age (GA) is presented in Table 1 .

Table 1. Relationship between ROP with GA

\begin{tabular}{|c|c|c|c|}
\hline $\begin{array}{c}\text { Total } \\
(\mathbf{N})\end{array}$ & $\begin{array}{c}\text { Without } \\
\text { ROP } \\
\text { N (\%) }\end{array}$ & $\begin{array}{c}\text { With ROP } \\
\text { N (\%) }\end{array}$ & $\begin{array}{c}\text { Gestational } \\
\text { age } \\
(\mathbf{G A})\end{array}$ \\
\hline $7 \%$ & $2(2 \%)$ & $5(5 \%)$ & $25-28$ \\
\hline $55 \%$ & $31(31 \%)$ & $24(24 \%)$ & $29-32$ \\
\hline $38 \%$ & $28(28 \%)$ & $10(10 \%)$ & $>32$ \\
\hline
\end{tabular}

17 cases of 39 infants with ROP (43. 5\%) had a birth weight of less than 1500 grams. The relationship of ROP with birth weight (BW) is presented in Table 2.

Table2. Relationship between ROP with BW

\begin{tabular}{|c|c|c|c|}
\hline $\begin{array}{c}\text { Total } \\
\text { (N) }\end{array}$ & $\begin{array}{c}\text { Without } \\
\text { ROP } \\
\text { N (\%) }\end{array}$ & $\begin{array}{c}\text { With ROP } \\
\text { N (\%) }\end{array}$ & Weight \\
\hline $7 \%$ & $1 \%$ & $6 \%$ & $500-1000$ \\
\hline $29 \%$ & $16 \%$ & $13 \%$ & $1001-1500$ \\
\hline $43 \%$ & $30 \%$ & $13 \%$ & $1501-2000$ \\
\hline $21 \%$ & $14 \%$ & $7 \%$ & $>2000$ \\
\hline
\end{tabular}

In the study of correlation of variables with ROP using the single-variable logistic regression model, the following results were obtained: based on the findings, the age of less than 32 weeks $(\mathrm{OR}=2$. 91, $\mathrm{P}$-value $=0$. $017)$, birth weight (BW) less than 1500 grams $(\mathrm{OR}=3$. 512, $\mathrm{P}$-value $=0$. 007) and sepsis $(\mathrm{OR}=2.88, \mathrm{P}$-value $=0$. 046) were significantly associated with ROP. Six infants from the study group underwent blood transfusion, 2 of them had no ROP, and 4infants had ROP, which did not show any significant correlation between blood transfusion and ROP. (P-value=0. 20)The mean duration of oxygen therapy in with retinopathy group was $6.1 \pm 5$. 7 and in without retinopathy group was 3. 48 \pm 3.36 days, which had a significant correlation with ROP. (Pvalue $=0.012$ ). The mean duration of admission in with retinopathy group was $24.59 \pm 17.01$ and in without retinopathy group was $15.02 \pm 9.63$ days, which was related to ROP $(\mathrm{P}-$ value $=0.002)$.

\section{Discussion and Conclusion}

In this study, from 100 samples with a mean GA of $31.83 \pm 2.06$ weeks and a mean BW of $1686.9 \pm 416$. 46 grams, Retinopathy of Prematurity was observed in $39 \%$ of cases. Severe ROP types require treatment in 14 infants from 39 preterm infants with retinopathy (35. $8 \%$ ). Among cases with retinopathy, $20.5 \%$ were at stage $1,43.5 \%$ at stage 2 and $36 \%$ at stage 3 disease.

Mousavi et al. , during the years 2003-2007, with a study of 1053 premature infants who referred to Farabi Hospital in Tehran, reported an ROP of 36. 1\% (19). Karkhaneh et al. , also conducted a study on 953 premature infants in Tehran from 2003 to 2007, with an overall prevalence of ROP of $34.4 \%$ and a severe type of ROP 22. 6\% (20).

Multu et al. , in Turkey, evaluated 314 premature infants with GA (less than 34 weeks), the prevalence of ROP was $37.1 \%$, of which $7.2 \%$ were at stage 3 or above, in $16.1 \%$ of cases, treatment for premature retinopathy was required (21). The results of these studies were similar to those of the present study in terms of prevalence of ROP.

In a survey conducted in New York by Chiang et al. during the years 1996-2000, among 1167427 premature infants, $0.2 \%$ or 1 in 511 infants suffered from ROP and about $9.5 \%$ of the samples were treated with laser (22). Larsson reported a general outbreak of ROP $25.5 \%$ in a study in infants less than 32 weeks of gestational age during 1998-2000(23). Ahmed, in Bangladesh, with a study of 114 infants during the years 1998-2003, reported an ROP of 4. 4\% (24). In the study of Shah et al in Singapore, among 564 premature infants, $29.2 \%$ of cases had premature retinopathy, $49 \%$ had stage 1 disease, $24 \%$ had stage 2 and $7 \%$ had stage 3 or higher, and $62.2 \%$ had need of treatment (25).

According to studies, this wide difference in the prevalence of ROP in various studies in different countries is probably due to the difference in the rate of progression of the NICU and, consequently, the survival of infants with birth weight and gestational age (Using pre-natal steroid injections to mother as well as surfactant prophylaxis for the development of neonate lung maturity). Considering that low birth weight and low gestational age are the most important risk factors for retinopathy, the best way to prevent the development of ROP is to prevent premature infant births, but reducing risk factors along with this problem is effective in reducing its incidence. With the proper management of oxygen therapy, the control of infections in the NICU segment to prevent sepsis and control other risk factors can reduce the incidence of retinopathy. Therefore, the treatment teams need to pay more attention to these issues.

In a study of 39 infants with preterm retinopathy, 14 infants had severe ROP types requiring therapy. All 14 infants with severe ROP underwent intravitreal injection, with good visual prognosis in all 14 cases, and no case needed to be re-treated, also, none of the samples progressed to the higher stages of the disease. 25 infants with mild ROP from 39 infants with retinopathy (64. 1\%) had spontaneous regression without need for treatment. Based on the present study, the prevalence of retinopathy is high, which can be indicative of the improvement of NICU and the survival of premature infants (birth weight and low gestational age). Also, preventing the birth of a premature infant and correcting oxygen therapy is a major contributor to Retinopathy of Prematurity and the optimal prognosis after treatment indicates the importance of timely diagnosis and treatment of ROP, which delay in examinations after the 6th week of birth and delayed treatment, can have serious consequences for the sight of the infant. Therefore, considering the risk of premature infants, routine screening for all NICU sections of the infant is recommended. 


\section{Suggestions}

One of the research limitations is the difficulty of conducting examinations in preterm infants due to the lack of co-operation of the patients during the examination, full pupil dilation and examination of all parts of the retina, in particular the prefill part, for examination of vascularization, as well as the lack of regular referral of patients due to the lack of awareness of their families about the importance of conducting regular and sequential examinations to determine progression or improvement of the disease. The following suggestions are made to make work better:

Design a specific screening plan for physicians and staff members of the premature infants who do not have enough knowledge in this area.

The Ministry of Health's Healthcare Program requires more support by raising the level of public awareness and NICU staff.

If the infant is at risk and the family is not suitable, conditions will be created to inform the infant by the hospital staff to the relevant centers.

The need for parents to know about retinopathy.

\section{References}

1. Dale Ph. Retinopathy of Prematurity In: Avroy A . Far-off, Richard J .Martin: Neonatal-Perinatal medicine diseases of the fetus and Infant8 .thedition . Mosby, London 2006; 1747- .1754

2. Gilbert C, Foster A. Childhood blindness in the context of VISION 2020-the right to sight. Bull World Health Organ 2001; 79(3):227-32.

3. Clemett R, Darlow B. Results of screening low-birth -weight infants for retinopathy of prematurity. Curr Opin Ophthalmol 1999; 10(3):155-63.

4. Saugstad OD. Oxygen and retinopathy of prematurity. J Perinatol 2006; 26 Suppl: S46-50.

5. Gilbert C, Rahi J, Eckstein M .Retinopathy of prematurity in middle-income countries. Lancet . 1997; 350:12 - .14

6. Scott KE, Kim DY, Wang L, Kane SA, Coki O, Starren J, et al. Tele medical diagnosis of retinopathy of prematurity intra physician agreement between ophthalmoscopy examination and imagebased interpretation .Ophthalmology 2008; 115:1222- .1228

7. Gibson DL, Sheps SB, Uh SH, Schechter MT, and McCormick AQ: Retinopathy of prematurityinduced blindness: birth weight-specific survival and the new epidemic. Pediatrics 1990; 86:405-12.

8. Avery GB, Glass P: Retinopathy of prematurity: progress report. Pediatr Ann 1988; 27 $\square 327 . \therefore, 520$ $532,530-.3$

9. Lermann VL, Fortes Filho JB, Procianoy RS .The prevalence of retinopathy of prematurity in very low birth weight newborn .J Pediatr (Rio J))82;2006 . (1: .32-27

10. Brown BA, Thach AB, Song JC, Marx JL, Kwun RC, Frambach DA .Retinopathy of prematurity: Evaluation of risk factor. Int Ophthalmol 1998; 22: 279-.283
11. Seiberth V, Linderkamp O .Risk factors in retinopathy of prematurity .A multivariate statistical analysis .Ophthalmological 2000; 214:131 - .135

12. Wallace DK, Kylstra JA, Phillips SJ, Hall JG .Poor postnatal weight gain: A risk factor for severe retinopathy of prematurity .J AAPOS;2000 . 4:343.347

13. Palmer EA, Flynn JT, Hardy RJ, et al. Incidence and early course of retinopathy of prematurity. The Cryotherapy for Retinopathy of Prematurity Cooperative Group. Ophthalmology. 1991; 98:1628.

14. Gilbert C. Retinopathy of prematurity: a global perspective of the epidemics, population of babies at risk and implications for control. Early Hum Dev 2008; 84 (2):77-82.

15. Screening examination of premature infants for retinopathy of prematurity. Pediatrics 2006; 117:572.

16. Clarke WN, Hodges E, Noel LP, et al. The oculocardiac reflex during ophthalmoscopy in premature infants. Am J Ophthalmol 1985; 99: 649.

17. Multicenter trial of cryotherapy for retinopathy of prematurity. Three-month outcome. Cryotherapy for Retinopathy of Prematurity Cooperative Group. Arch Ophthalmol 1990; 108:195.

18. Reynolds JD, Hardy RJ, Kennedy KA, et al. Lack of efficacy of light reduction in preventing retinopathy of prematurity. Light Reduction in Retinopathy of Prematurity (LIGHT-ROP) Cooperative Group. N Engl J Med 1998; 338:1572.

19. Mousavi SZ, Riazi Esfahani M, Roohipoor R ,et al. Characteristics of Advanced Stages of Retinopathy of Prematurity. Iranian Journal of Ophthalmology 2010; 22(2):19-24.

20. Karkhaneh R, Mousavi SZ, Riazi-Esfahani M, et al. Incidence and risk factors of retinopathy of prematurity in a tertiary eye hospital in Tehran. Br J Ophthalmol 2008; 92:1446-9 .

21. Mutlu FM, Altinsoy HI, Mumcuoglu T, et al. Screening for retinopathy of prematurity in a tertiary care newborn unit in Turkey: frequency, outcomes, and risk factor analysis. J Pediatr Ophthalmol Strabismus. 2008 Sep-Oct; 45 (5):291-8.

22. Chiang MF, Arons RR, Flynn JT, et al. Incidence of retinopathy of prematurity from 1996 to 2000: analysis of a comprehensive New York state patient database. Ophthalmology. 2004 Jul; 111 (7):131725.

23. Larsson E, Carle-Petrelius B, Cernerud G, et al. Incidence Of Retinopathy Of Prematurity In Two Consecutive Swedish Population Base Studies. Br J Ophthalmol 2002;86(100 ):1122-6 .

24. Ahmed AS, Muslima H, Anwar KS, et al. Retinopathy of prematurity in Bangladeshi neonates. J Trop Pediatr. 2008 Oct; 54(5):333-9. Epub 2008 May 25.

25. Shah VA, Yeo CL, Ling YLF, et al. Incidence, Risk Factors of Retinopathy of Prematurity Among Very Low Birth Weight Infants in Singapore. Ann Acad Med Singapore 2005; 34:169-78. 